\title{
Study on Maternal SNPs of MTHFR Gene and HCY Level Related to Congenital Heart Diseases
}

\author{
Hui Shi ${ }^{1}$ (D) Shiwei Yang ${ }^{2} \cdot$ Ning Lin $^{1} \cdot$ Peng Huang ${ }^{3} \cdot{\text { Rongbin } \mathrm{Yu}^{3} \cdot \text { Mei Chen }}^{2} \cdot$ Lijuan Wang $^{1} \cdot$ Zhixin Jiang $^{1}$. \\ Xiaoru Sun ${ }^{1}$
}

Received: 17 April 2020 / Accepted: 30 August 2020 / Published online: 21 November 2020

(C) The Author(s) 2020

\begin{abstract}
The aim of this study is to evaluate the relationship between maternal single nucleotide polymorphisms (SNPs) of methylenetetrahydrofolate reductase (MTHFR) gene with plasma homocysteine (HCY) level and offspring congenital heart diseases (CHDs). 338 mothers with offspring CHDs as case group and 306 mothers of normal children as control group were recruited. Their pregnant histories were interviewed by questionnaire and the MTHFR rs1801133 and rs1801131 were genotyped. The case-control analysis was used to find out the relationship between maternal SNPs of MTHFR gene and offspring CHDs. And the plasma HCY concentration of the mothers of CHDs children was detected. This case-case study was intended to find out the relevance between maternal HCY level and SNPs of MTHFR gene. There were significant differences in the gender of children, occupation of mothers, family history with CHDs, history of abortion, history of adverse pregnancy, early pregnancy health, fetus during pregnancy, pesticide exposure and drug exposure in CHDs group and control group $(P<0.05)$. MTHFR rs 1801133 was significantly associated with their offspring CHDs in mothers. The polymorphism of maternal MTHFR rs 1801133 increased plasma HCY level, especially the homozygous mutation. Besides the environmental factors, our results suggested that the maternal MTHFR rs1801133 polymorphism might be a risk factor of their offspring CHDs, which may be due to the hyperhomocysteinemia by abnormal metabolism of HCY.
\end{abstract}

Keywords Congenital heart disease $\cdot$ SNPs of MTHFR $\cdot$ Homocysteine

\section{Introduction}

Congenital heart diseases (CHDs) refers to abnormal cardiovascular structure or function at birth. Monitoring data on birth defects in China showed that CHDs have been the highest incidence of birth defects and increased yearly since 2005 [1]. They became the first cause of death among 1 year

Hui Shi and Shiwei Yang have contributed equally to this work.

Electronic supplementary material The online version of this article (https://doi.org/10.1007/s00246-020-02449-1) contains supplementary material, which is available to authorized users.

Xiaoru Sun

sunxr@nju.edu.cn

1 Jiangsu Institute of Planned Parenthood Research, Nanjing 210036, Jiangsu, China

2 Department of Cardiology, Children's Hospital of Nanjing Medical University, Nanjing 210008, China

3 Nanjing Medical University, Nanjing 210029, Jiangsu, China infants in China due to severe illness, multiple complications and high mortality [2]. The etiology of CHDs is not fully understood. A great amount of studies indicated CHDs are affected by both genetic and environmental factors [3, 4]. Methylenetetrahydrofolate reductase (MTHFR), the major enzymes in the folate/homocysteine (HCY) metabolism pathway, catalyzes the conversion of 5,10-methylenetetrahydrofolate into 5-methyltetrahydrofolate. The mutation of the MTHFR gene results in the decrease of the enzyme activity and the synthesis of methionine, the direct precursor of $S$-adenosylmethionine (SAM) which is an important methyl donor in cells participating in the tissue development and growth. Most common genetic variants of the MTHFR gene are the $677 \mathrm{C}>\mathrm{T}$ polymorphism (rs1801133) and 1298A $>\mathrm{C}$ polymorphism (rs1801131), which result in the conversion of alanine to valine and glutamate to alanine, respectively. In our previous study, we found environment risks for CHDs in Chinese and the maternal genotype in MTHFR rs1801131 variant significantly increased offspring CHDs risk by screened five single nucleotide polymorphisms (SNPs) 
of three genes [5]. However, the relationship between the maternal plasma HCY level and SNPs of MTHFR was only showed a tendency without statistic significances. The objective of the present further study was to evaluate the relationship between the genotype and HCY level in the plasma of mothers with offspring CHDs by case-case study in a larger crowd, and meanwhile a further investigation on maternal environmental risks and the genotype factor in MTHFR by case-control study.

\section{Materials and Methods}

\section{Ethics Statement}

The study had been approved by the Ethics Review Committee of the Jiangsu Institute of Planned Parenthood Research, Nanjing, China. Prior written informed consent was obtained from all the participants enrolled in the study.

\section{Participants}

From May 2012 to September 2016, the mothers of CHDs children in the study were recruited from the Nanjing Children's Hospital, and the mothers of healthy children with matching age were recruited from the preconception health care center, Jiangsu Province, China. They were interviewed face-to-face to collect personal information and excluded diseases such as CHDs, hypertension, diabetes, tumor and others. Each participant donated $3 \mathrm{~mL}$ venous blood for $\mathrm{HCY}$ tests and host DNA genotyping.

\section{DNA Collection and Genotyping}

Genomic DNA was extracted from a leukocyte pellet by traditional proteinase $\mathrm{K}$ digestion and followed by phenol-chloroform extraction and ethanol precipitation. MTHFR rs1801133 and rs1801131 were genotyped by the Taq-Man allelic discrimination assay on an ABI 7900 system (Applied Biosystems, La Jolla, CA). The information on primers and probes are shown in Table $\mathrm{S}$.

\section{Detection of HCY}

Plasma was separated from venous blood with EDTA anticoagulant in time. HCY tests were performed by circulatory enzyme method on Backman automatic biochemical analyzer in Clinical Laboratory of Zhongda hospital southeast university. The HCY detection were performed according to the manufacturer's (NingBo Medical System Biotechnology Co., Ltd) protocols briefly as follows. The sample was mixed with reagent 1 and incubated at $37{ }^{\circ} \mathrm{C}$ for $5 \mathrm{~min}$. Then the mixture was added reagent 2 , and incubated at $37{ }^{\circ} \mathrm{C}$ for another $2 \mathrm{~min}$. The absorbance change of the sample was monitored for 3 min under the detection wavelength of $340 \mathrm{~nm}$ (main) $/ 405 \mathrm{~nm}$ (auxiliary), and the HCY concentration was calculated by the formula.

\section{Statistical Analysis}

The survey data were recorded independently by two individuals and were checked after entry. Counting variables were described by frequency (percentage), and their differences between groups were tested by $\chi^{2}$ test. Quantitative data were described by means \pm standard deviation $($ mean $\pm \mathrm{SD})$, their difference between groups was tested by $t$-test or One-Way ANOVA. We used median $(25 \%$ and $75 \%$ interquartile) to describe skewness distribution variables, and their difference between groups was tested by rank sum test. Odds ratios (ORs) and their 95\% confidential intervals (CIs) were calculated as a measure of difference in the response rate using logistic regression analysis. Multivariate logistic regression was used to analysis the association of selected SNPs with offspring CHDs, in which factors showing significant differences in univariate analysis were included for adjustment.

The statistical analyses were performed using Statistical Analysis System software (version 9.1.3, SAS Institute, Cary, NC). All $P<0.05$ in a two-sided test was considered statistically significant.

\section{Results}

In this study, a total of 338 mothers with offspring CHDs and 306 mothers of normal children with available blood sample were included. The selected characteristics of the mothers in CHDs group and the controls in the study are shown in Table 1. There were significant differences in the gender of children, child-bearing age, education, occupation, history of abortion, early pregnancy health, smoking, pesticide exposure and drug exposure in two groups $(P<0.05$ for both comparisons).

The genotype percentage values for MTHFR rs 1801133 and rs 1801131 have been given in Table 2 for the mothers. All the distributions were found to be in agreement with Hardy-Weinberg equilibrium. Logistic regression analyses showed that MTHFR rs1801133 was significantly increased their offspring CHDs risk compared with control group of mothers (OR 6.24, 95\% CI 2.87-13.56 in Recessive model) (Table 2).

Table 3 shows the association of MTHFR rs1801133 and rs1801131 with plasma HCY level in mothers with offspring CHDs (12 unqualified samples of venous blood 
Table 1 Demographic and selected variables in mothers with offspring CHDs and controls

\begin{tabular}{|c|c|c|c|}
\hline Variables & Control $N(\%)$ & Case $N(\%)$ & $P$ \\
\hline Gender of children $^{\mathrm{a}}$ & & & $<0.001$ \\
\hline Male & $171(57.00)$ & $138(40.95)$ & \\
\hline Female & $129(43.00$ & 199(59.05) & \\
\hline \multicolumn{4}{|l|}{ Child-bearing age $\mathrm{e}^{\mathrm{a}, \mathrm{b}}$} \\
\hline $24.12 \pm 5.11$ & $25.93 \pm 2.24$ & $26.52 \pm 5.25$ & 0.075 \\
\hline$<25$ & $90(31.25)$ & $150(45.18)$ & $<0.001$ \\
\hline$\geq 25$ & $198(68.75)$ & $182(54.82)$ & \\
\hline Education $^{\mathrm{a}}$ & & & $<0.001$ \\
\hline Middle school and lower & $110(39.71)$ & $192(56.97)$ & \\
\hline High school and above & $167(60.29)$ & $145(43.03)$ & \\
\hline Occupation $^{\mathrm{a}}$ & & & 0.005 \\
\hline Farmer & $44(15.83)$ & $66(19.94)$ & \\
\hline Worker & $76(27.34)$ & $80(24.17)$ & \\
\hline Server & $15(5.40)$ & $34(10.27)$ & \\
\hline Businessman & 25(8.99) & $28(8.46)$ & \\
\hline Housework & $35(12.59)$ & $356(10.57)$ & \\
\hline Teacher/government worker & $28(10.07)$ & $11(3.32)$ & \\
\hline Others & $55(19.78)$ & $77(23.26)$ & \\
\hline Family history with CHDs ${ }^{\mathrm{a}}$ & & & 0.118 \\
\hline No & $272(97.84)$ & $321(95.54)$ & \\
\hline Yes & $6(2.16)$ & $15(4.46)$ & \\
\hline History of abortion ${ }^{\mathrm{a}}$ & & & 0.025 \\
\hline No & $185(66.55)$ & 194(57.74) & \\
\hline Yes & $93(33.45)$ & $142(42.26)$ & \\
\hline History of adverse pregnancy ${ }^{\mathrm{a}}$ & & & 0.67 \\
\hline No & $127(45.68)$ & $156(47.42)$ & \\
\hline Yes & $151(54.32)$ & $173(52.58)$ & \\
\hline Early pregnancy health ${ }^{\mathrm{a}}$ & & & $<0.001$ \\
\hline Healthy & 242(88.32) & 197(60.80) & \\
\hline Illness & $32(11.68)$ & $127(39.20)$ & \\
\hline Fetus during pregnancy ${ }^{\mathrm{a}}$ & & & 0.136 \\
\hline Normal & 266(97.08) & $317(94.63)$ & \\
\hline Abnormal & $8(2.92)$ & $18(5.37)$ & \\
\hline Folate supplements or multiple vitamin ${ }^{\mathrm{a}}$ & & & 0.277 \\
\hline No & $130(42.48)$ & $158(46.75)$ & \\
\hline Yes & $176(57.52)$ & $180(53.25)$ & \\
\hline Smoking ${ }^{\mathrm{a}}$ & & & 0.022 \\
\hline No & 173(83.17) & 302(89.88) & \\
\hline Yes & $35(16.83)$ & $34(14.58)$ & \\
\hline Drinking $^{\mathrm{a}}$ & & & 0.167 \\
\hline No & 254(92.70) & 297(89.46) & \\
\hline Yes & $20(7.30)$ & $35(10.54)$ & \\
\hline Pesticide exposure $^{\mathrm{a}}$ & & & 0.023 \\
\hline No & 269(98.53) & $319(95.22)$ & \\
\hline Yes & $4(1.47)$ & $16(4.78)$ & \\
\hline Drug exposure $^{\mathrm{a}}$ & & & $<0.001$ \\
\hline No & 244(90.37) & 247(75.77) & \\
\hline Yes & $26(9.63)$ & $79(24.23)$ & \\
\hline
\end{tabular}

${ }^{\mathrm{a}}$ Counting variables were described by frequency (percentage), and tested by $\chi^{2}$ test for differences between groups

${ }^{b}$ Quantitative data were described by mean $\pm S D$, and tested by t-test for differences between groups 
Table 2 Association of selected SNPs with offspring CHDs in mothers

\begin{tabular}{lllll}
\hline Genotype & Control $N(\%)$ & Case $N(\%)$ & OR $(95 \% \text { CI })^{\mathrm{a}}$ & $P$ value $^{\mathrm{a}}$ \\
\hline rs1801133 & & & & \\
CC & $94(30.72)$ & $108(31.95)$ & 1 & \\
CT & $159(51.96)$ & $161(47.63)$ & $0.74(0.46-1.19)$ & 0.211 \\
TT & $53(17.32)$ & $69(20.41)$ & $5.22(2.29-11.91)$ & $<0.001$ \\
Dominant & & & $1.06(0.67-1.66)$ & 0.812 \\
Recessive & & & $6.24(2.87-13.56)$ & $<0.001$ \\
Additive & & & $1.59(1.13-2.22)$ & 0.007 \\
rs1801131 & & & & \\
AA & $207(67.65)$ & $228(67.46)$ & 1 & \\
AC & $92(30.07)$ & $91(26.92)$ & $1.00(0.62-1.62)$ & 0.996 \\
CC & $7(2.29)$ & $19(5.62)$ & $2.18(0.74-6.40)$ & 0.157 \\
Dominant & & & $1.12(0.71-1.78)$ & 0.624 \\
Recessive & & & $2.17(0.77-6.32)$ & 0.155 \\
Additive & & & $1.19(0.82-1.74)$ & 0.36 \\
\hline
\end{tabular}

${ }^{a}$ Adjusted by gender of children, child-bearing age group, education, occupation, history of abortion, early pregnancy health, smoking, pesticide exposure an drug exposure

Table 3 Association of selected SNPs with plasma HCY level in mothers with offspring CHDs

\begin{tabular}{lclll}
\hline Variables & $N$ & Median & $95 \%$ CI & $P$ \\
\hline rs1801133 & & & & $<0.001$ \\
CC & 103 & 8.4 & $5.88-10.92$ & \\
CT & 158 & 9.35 & $5.61-13.09$ & \\
TT & 65 & 11.5 & $5.88-17.12$ & \\
rs1801133 & & & & $<0.001$ \\
CC & 103 & 8.4 & $5.88-10.92$ & \\
CT/TT & 223 & 9.8 & $5.26-14.34$ & \\
rs1801133 & & & & \\
CC/CT & 261 & 9 & $5.65-12.33$ & \\
TT & 65 & 11.5 & $5.88-17.12$ & \\
rs1801131 & & & & \\
AA & 221 & 9.3 & $4.98-13.62$ & \\
AC & 88 & 9.8 & $6.14-13.46$ & \\
CC & 17 & 9.2 & $6.41-11.98$ & \\
rs1801131 & & & & 0.691 \\
AA & 221 & 9.3 & $4.98-13.62$ & \\
AC/CC & 105 & 9.5 & $5.97-10.03$ & \\
rs1801131 & & & & $5.36-13.64$ \\
AA/AC & 309 & 9.5 & $6.41-11.99$ & \\
CC & 17 & 9.2 & & \\
\hline
\end{tabular}

were excluded). The polymorphism of maternal MTHFR rs1801133 significantly increased plasma HCY level, especially with the homozygous mutation.

\section{Discussion}

The critical period of embryonic heart development is at 3-8 weeks of gestation. Environmental factors, as important as genetic factors, are causes of CHDs which mainly include the living environment, infection factors and drug effects of pregnant women [6-9]. In this study we found that gender of children, mothers' child-bearing age, education, occupation, history of abortion, early pregnancy health, smoking, pesticide exposure and drug exposure were significantly associated with the risk of offspring CHDs susceptibility. The findings are consistent with those we observed in previous study.

MTHFR gene, catalyzes the conversion of 5,10-methylenetetrahydrofolate into 5-methyltetrahydrofolate. The latter is involved in the transformation of HCY to methionine, releasing tetrahydrofolate, which is decreasing the level of plasma HCY [10]. Therefore, the correlation between polymorphism of MTHFR gene and CHDs has become research focuses [11-13]. However, there is a great controversy reports about the relationship between the maternal MTHFR rsl801133 and rsl801131 polymorphism and their offspring CHDs [12, 14-16]. Some of them considered that rs1801133 and rs1801131 were related with the risk of CHDs [12,13]. But some studies suggested that there is no association between the parents' polymorphism of MTHFR rs1801133 and children' CHDs [14]. Even there was a report identified MTHFR rs1801131 as a protective locus against CHDs [17]. In our previous study, we found the mothers who got a mutation of MTHFR rs1801131 CC genotypes had a $267 \%$ increase in risk of given birth of a CHDs children (OR 3.67, 95\%CI 1.12-12.05). But in this study we found that the rs1801133 variant genotypes significantly increased their offspring CHDs risk by $524 \%$ (OR 6.24, 95\% CI 2.87-13.56 in Recessive model) while no statistic difference between MTHFR rs1801131 polymorphism and offspring CHDs. The inconsistency of our results may be due to different adjustment variables, in the current study we additionally adjusted child-bearing age, education and smoking without adjusting family history with CHD, history of adverse pregnancy and fetus during pregnancy. Therefore, a larger sample size studies are required to achieve more robust results.

The plasma HCY level is another tricky problem. It was affected both by genetic and nutritional elements. In our previous study, we found that it was associated with offspring CHDs, and the critical value was $8.9 \mu \mathrm{mol} / \mathrm{L}$ determined by the ROC curve [18]. It is proved by nutrition studies that folic acid was key methyl donor in the plasma HCY metabolic pathway and VitB12 participates in the transfer of it. HCY could not be methylated to methionine by deficiency of folic acid or VitB12, which 
leads to hyperhomocysteinemia. However, the relationship between MTHFR rs1801133 and plasma HCY level were not very clear. It is reported that the polymorphism of MTHFR rs1801133 does not influence the level of plasma HCY. The mutation of MTHFR rs1801133 CT and TT genotypes is not found to be related to hyperhomocysteinemia [19] unless there is low level of folate occurred. In this study, we found that the polymorphism of maternal MTHFR rs1801133 significantly increased plasma HCY level, especially with the homozygous mutation, under the situation which there is no significant difference between the groups taking or not taking additional folate and multiple vitamins. We doubt that our crowd might not be lack of folate in Jiangsu Province, the south of China [20]. Thus, we come to the conclusion that the maternal MTHFR rs 1801133 polymorphism might be a risk factor of offspring CHDs due to the hyperhomocysteinemia by abnormal metabolism of HCY.

In summary, besides the environmental factors, the maternal MTHFR rs1801133 and rs1801131 polymorphisms might be risk factors of offspring CHDs in different pathway which require further investigation in a large quantity of populations.

Acknowledgements This study was funded by Maternal and Child Health Research Project of Jiangsu Provincial Commission of Health and Family Planning (Grant No. F201501), Scientific Research Project of Jiangsu Provincial Commission of Health and Family Planning (Grant No. Z2015-07), the National Natural Science Foundation of China (Grant No. 81670284), Nanjing Science and Technology Project (Grant No. 201715057).

\section{Compliance with Ethical Standards}

Conflict of Interest The authors declared that they have no conflicts of interest to this work.

Open Access This article is licensed under a Creative Commons Attribution 4.0 International License, which permits use, sharing, adaptation, distribution and reproduction in any medium or format, as long as you give appropriate credit to the original author(s) and the source, provide a link to the Creative Commons licence, and indicate if changes were made. The images or other third party material in this article are included in the article's Creative Commons licence, unless indicated otherwise in a credit line to the material. If material is not included in the article's Creative Commons licence and your intended use is not permitted by statutory regulation or exceeds the permitted use, you will need to obtain permission directly from the copyright holder. To view a copy of this licence, visit http://creativecommons.org/licenses/by/4.0/.

\section{References}

1. National Maternal and Child Health Monitoring and Annual Newsletter, 2016, 4. https://www.mchscn.org/Article_Show. asp?ArticleID $=637$

2. National Health and Family Planning Commission (2015) 2015 China yearbook of health and family planning statistics. Peking Union Medical College Press, Beijing
3. Zaidi S, Brueckner M (2017) Genetics and genomics of congenital heart disease. Circ Res 120(6):923-940

4. Blue GM, Kirk EP, Sholler GF et al (2012) Congenital heart disease: current knowledge about causes and inheritance. Med J Aust 197(3):155-159

5. Shi H, Yang S, Liu Y, Huang P, Lin N, Sun X, Yu R, Zhang Y, Qin Y, Wang L (2015) Study on environmental causes and SNPs of MTHFR, MS and CBS genes related to congenital heart disease. PLoS ONE 10(6):e0128646

6. Loffredo CA, Silbergeld EK, Ferencz C et al (2001) Association of transposition of the great ateries in infants with maternal exposures to herbicides and rodenticides. Am J Epidemiol 153(6):529-536

7. Karatza AA, Giannakopoulos I, Dassios TG et al (2011) Periconceptional tobacco smoking and isolated congenital heart defects in neonatal period. Int J Cardiol 148(3):295-299

8. Xiaojin L, Shusen Li, Lianzhong W et al (2002) Epidemiology analysis of correlative factor proportion of 2356 cases of congenital heart disease. Southwest Natl Def Med 12(4):298-301

9. Mengjie C, Meiying L, Qiuyan P et al (2012) Case-control study of maternal risk factor for fetel congenital heart diseases. Chin J Perinat Med 15(4):211-216

10. Kumar A, Palfrey HA, Pathak R, Kadowitz PJ, Gettys TW, Murthy SN (2017) The metabolism and significance of homocysteine in nutrition and health. Nutr Metab (Lond) 14:78

11. Zhang Q, Zha D, Dong P et al (2014) Association analysis between MTHFR genetic polymorphisms and the risk of congenital heart diseases in Chinese Han population. J Pharm Pharmacol 66(9):1259-1264

12. Huang J, Mei J, Jiang L et al (2014) MTHFR rs1801133 C>T polymorphism is associated with an increased risk of tetralogy of Fallot. Biomed Rep 2(2):172-176

13. Yu D, Zhuang Z, Wen Z et al (2017) MTHFR A1298C polymorphisms reduce the risk of congenital heart defects: a meta-analysis from 16 case-control studies. Ital J Pediatr 43(1):108

14. Mamasoula C, Prentice RR, Pierscionek T et al (2013) Association between C677T polymorphism of methylenetetrahydrofolate reductase and congenital heart disease: meta-analysis of 7697 cases and 13,125 controls. Circ Cardiovasc Genet 6(4):347-353

15. Li Z, Jun Y, Zhong-Bao R et al (2015) Association between MTHFR C677T polymorphism and congenital heart disease. A family-based meta-analysis. Herz 40(Suppl 2):160-167

16. Jiang Y, Mei J, Zhang W, Qian X, Zhang S, Liu C, Yang H (2015) Correlation between offspring congenital heart disease and MTHFR $677 \mathrm{C} / \mathrm{T}$ polymorphism and general status of pregnant women. Zhonghua Liu Xing Bing Xue Za Zhi 36(10):1072-1076

17. Hobbs CA, James SJ, Parsian A et al (2006) Congenital heart defects and genetic variants in the methylenetetrahydroflate reductase gene. J Med Genet 43(2):162-166

18. Shi H, Yang S, Sun X et al (2018) Study on risk value of homocysteine in pre-pregnancy women related to congenital heart disease. J Southeast Univ (Med Sci Ed) 37(4):593-596

19. Manetti A, Pollini I, Cecchi F et al (2000) The epidemiology of cardiovascular malformations. Genet Epidemiol 11:224

20. Hao L, Ma J, Stampfer MJ, Ren A, Tian Y, Tang Y, Willett WC, Li Z (2003) Geographical, seasonal and gender differences in folate status among Chinese adults. J Nutr 133(11):3630-3635

Publisher's Note Springer Nature remains neutral with regard to jurisdictional claims in published maps and institutional affiliations. 